\title{
Renewable Energy Sources and Energy Efficiency in Albania
}

\author{
Dott. Skerdilajd Bajramaj
}

Company KESH sh.a.

Email: skerdibajramaj@yahoo.com

\section{Doi:10.5901/ajis.2016.v5n3p179}

\begin{abstract}
The purpose of this paper is to underline the scientific potential of Albania in the field of RES and EE and show through a survey and analysis, concrete ways and means on how to fulfill in general the obligations arising from the signing of the Energy Community Treaty and the Third Package Directives: 2009/72/EC and 2009/73/EC, and also in compliance with the EU Directive 2009/28/EC "On the enhancement of the use of energy from renewable resources" and Directive 2012/27/EU of the European Parliament and of the Council of 25 October 2012 on energy efficiency, amending Directives 2009/125/EC and 2010/30/EU and repealing in particular the Directives 2004/8/EC and 2006/32/EC Text with the EEA relevance. The transpose of European Union legislation by national legislation and the reform that has accompanied it have significantly improved some of the key indicators. The results arising from a statistical and empirical study are in general very encouraging for the future of the energy sector. Also, the situation in which we are and the measures taken so far make us optimistic that Albania will fulfill due obligations towards international institutions in particular to the field of renewable energy and energy efficiency.
\end{abstract}

Keywords: Target, Energy, Renewable, Efficiency.

\section{Introduzione}

In Albania, studi e lavori scientifici credibili ed importanti sono carenti o mancano del tutto a causa della scarsità di dati certi in quanto le diverse fonti offrivano dati differenti e spesso inerenti e non veritieri nella realtà Albanese. Non vi era presente, per motivi di organizzazione amministrativa, uno studio specifico per questo ho iniziato una ricerca mai avviata prima. La mia posizione lavorativa mi permette di avere accesso a dei dati interni amministrativi grazie ai quali ho iniziato lo studio. Mi sono confrontato con dei colleghi esperti di altri enti e istituzioni statali e ho estrapolato delle risorse non utilizzate adeguatamente allo scopo. Questo motivo mi ha spinto di porre delle domande e dei quesiti ai quali ho risposto e ho spiegato nell'articolo sottostante. Dunque, ritengo che lo studio che ho avviato dia un contributo riguardanti l'ambito dell'energia rinnovabile e la sua efficienza. Mi sono basato molto sulle direttive e le raccomandazioni che ha dato l'Unione Europea sul tema in questione e ho redatto l'articolo tenendo conto dei passi che può fare l'Albania in merito.

La mitigazione dei cambiamenti climatici gioca un ruolo importante nella strategia energetica dell'UE, principalmente grazie alla rilevanza data all'efficienza energetica e alle fonti di energie rinnovabili. In particolare sono considerati due orizzonti, di breve e medio periodo, il 2020 e il 2030. Entro il 2020 l'UE mira a soddisfare il 20\% del suo fabbisogno energetico da fonti rinnovabili e realizzare un aumento del $20 \%$ dell'efficienza energetica. Entro il 2030, I'UE mira a "una riduzione del 40\% delle emissioni di gas ad effetto serra rispetto ai livelli del 1990; almeno una quota del 27\% del consumo di energia rinnovabile; almeno il $27 \%$ di risparmio energetico secondo lo scenario previsto dal business-asusual".

L'efficienza della direttiva energetica 2012 (EED) è il maggior strumento usato dalla politica europea che delinea un quadro politico generale volto ad ottenere un risparmio energetico del 20\% entro il 202012 e stabilisce un quadro comune di misure a tal fine. La direttiva completa il quadro politico dell'UE in materia di efficienza energetica, già includendo il rendimento energetico dell'edilizia, l'Eco-design e l'etichettatura delle direttive Energy.

L'approccio politico EED è principalmente specificato nell'art.7, che dovrebbe fornire più della metà dei risparmi energetici richiesti. In particolare, è previsto che gli Stati membri (SM) introducano schemi obbligatori di efficienza energetica o misure politiche alternative volte a raggiungere un risparmio annuo del 1,5\% delle vendite di energia ai clienti finali di tutti i distributori di energia o le società di vendita al dettaglio. In attuazione di tale disposizione gli Stati membri possono scegliere tra una vasta gamma di misure politiche e azioni individuali volti al miglioramento dell'efficienza energetica.

Analogamente, la direttiva sulle energie rinnovabili del 2009 definisce una politica globale per la produzione e la 
promozione di energia da fonti rinnovabili nell'UE. Si richiede all'UE di soddisfare almeno il $20 \%$ del suo fabbisogno energetico totale con le fonti rinnovabili entro il 2020 - da conseguire attraverso il raggiungimento dei singoli obiettivi nazionali. Tutti i paesi dell'UE devono garantire che almeno il 10\% dei loro carburanti per il trasporto provenga da fonti rinnovabili, entro il 2020. Anche in questo caso, la direttiva prevede obiettivi nazionali di energia rinnovabile per ogni paese, tenendo conto del suo punto di partenza e il potenziale complessivo delle energie rinnovabili.

Gli Stati membri hanno ancora una volta il compito di escogitare un modo per raggiungere questi obiettivi e sono tenuti a specificare la linea generale della loro politica delle energie rinnovabili nei piani di azione nazionali di energie rinnovabili. La possibilità, data ai paesi membri, porta alla cooperazione per raggiungere le fonti energetiche rinnovabili (FER) obiettivi conquistati attraverso meccanismi di cooperazione, come i trasferimenti statistici di energia rinnovabile, insieme a progetti comuni di energia rinnovabili e regimi comuni di sostegno alle energie rinnovabili. Disposizioni specifiche sono fatte per i biocarburanti, in quanto sono gli strumenti che aiutano i paesi dell'UE a rispettare il loro 10\%, destinato alle energie rinnovabili nel settore dei trasporti, e devono rispondere ai criteri di sostenibilità per tutti i biocarburanti prodotti o consumati nella UE per garantire che essi siano prodotti in modo sostenibile e nel rispetto dell'ambiente.

II governo Albanese considera l'implementazione della promozione delle energie rinnovabili e dell'energia efficente come uno strumento importante delle politiche energetiche per aumentare la sicurezza dell'approvvigionamento energetico, dello sviluppo economico, della sostenibilità del settore energetico e della tutela dell'ambiente.

Inoltre, l'Albania come una delle parti contrattuali del trattato Comunitario dell'energia ha l'obbligo di recepire la direttiva UE 2009/28/CE "sulla valorizzazione dell'uso di energia da fonti rinnovabili" e di essere in conformità con essa. Una delle richieste della legge 138/2013 "sulle risorse energetiche rinnovabili" e della presente direttiva è la preparazione e l'approvazione del piano d'azione nazionale per le energie rinnovabili (NREAP), che stabilisce gli obiettivi nazionali di energie rinnovabili nel consumo domestico interno finale, nonché le misure di sostegno per raggiungere l'obiettivo.

Anche se l'Albania garantisce oltre il 90\% della potenza dalle stazioni idroelettriche, una parte considerevole dell'energia viene importata. Questa parte oscilla a seconda delle variazioni delle condizioni annuali - tra il $30 \%$ e il $60 \%$ della fornitura totale con il Prime Energy. Le energie rinnovabili potrebbero essere una soluzione per la riduzione di questa dipendenza dalle importazioni e potrebbero migliorare non solo la sicurezza per l'approvvigionamento di energia, ma anche la sicurezza macroeconomica e la politica del paese nel diminuire il deficit finanziario del paese.

Lo sviluppo delle FER è importante perché aiuta il paese a soddisfare almeno due dei suoi obiettivi strategici: la sicurezza dell'approvvigionamento energetico e la sostenibilità. L'Albania è un caso speciale perché la sua produzione di energia elettrica è realizzata in larga maggioranza con idroelettrici centrali di dimensioni medie-grandi. Inoltre, circa il 10$13 \%$ delle fonti di energia primaria totale (TPE) del paese - comprese le importazioni - sono forniti da biomassa, in particolar modo da legna da ardere per il fuoco.

Tuttavia, la dipendenza del paese dall'idroelettricità rende vulnerabili le variazioni delle condizioni idrologiche, come testimoniato durante i periodi di siccità, e questo ha ridotto drasticamente la sicurezza dell'alimentazione elettrica. A questo proposito, sono state fatte delle analisi molto dettagliate secondo la Prima ${ }^{1}$ e la Seconda ${ }^{2}$ comunicazione nazionale Albanese alla Convenzione Quadro delle Nazioni Unite sui Cambiamenti Climatici, sostenuti dal GEF/UNDP, per quanto riguarda gli effetti del cambiamento climatico sulla produzione di energia elettrica da hidrocentrali su fiumi Drini e Mati.

Anche se gli ultimi due anni $(2014,2015)$ hanno registrato condizioni idrologiche normali, la minaccia rimane. Un'altra difficoltà è il continuo ritardo della riabilitazione di idro-centrali che porta alla riduzione di disponibilità energetica. D'altra parte, l'Albania ha notevoli importazioni di energia che variano - a seconda delle condizioni annuali - tra il 25 e il 40\% di TPE.

L'energia rinnovabile può essere una soluzione per ridurre la dipendenza strategica dalle importazioni e migliorare non solo la sicurezza dell'approvvigionamento energetico, ma anche la protezione delle macro economie e la politica del paese, riducendo il deficit del bilancio del paese. Infine, lo sviluppo di progetti di energia rinnovabile attrae investimenti esteri e genera nuovi posti di lavoro per l'Albania.

Anche per l'Albania, dove oltre il $97 \%$ di energia elettrica e il $20-25 \%$ di fonti primarie totali sono forniti da fonti di acqua, l'uso di altre fonti rinnovabili è importante perché migliora la sicurezza dell'approvvigionamento energetico e della 
sostenibilità del settore energetico. Se aggiungiamo a questi la riduzione del deficit commerciale estero di un paese importatore netto - e Albania è un paese così - è ovvia l'importanza della riforma.

Durante la scorsa legislatura, il governo Albanese ha fatto importanti sforzi per migliorare il quadro normativo e legale per l'energia. In questa direzione, ha fatto diversi tentativi di cambiare le leggi esistenti e per incoraggiare una competizione nel campo delle produzioni di energia in generale e del RES in particolare. Insieme a una serie di miglioramenti della legge, sono state approvate quattro leggi fondamentali: la legge per le concessioni, per il settore energetico (approvata dal Parlamento Albanese nel maggio 2015), per le energie rinnovabili (progetto presentato per essere approvato dal Parlamento dell'Albania), per l'efficienza di energia nel campo edilizio (il progetto di legge è stato approvato l'anno precedente. E 'stato preparato in linea con la direttiva UE) .

Lo scopo di queste leggi è la liberalizzazione del mercato dell'energia, l'aumento della concorrenza, la promozione dello sviluppo delle risorse rinnovabili e l'attrazione degli investimenti esteri nel settore energetico e garantire una riforma sostenibile nel settore dell'energia. Per quanto riguarda lo sviluppo dei progetti di energia rinnovabile, il processo di autorizzazione è stato migliorato. L'istituzione di un ufficio di uno sportello unico per le licenze è un grande passo in avanti fatto dal governo Albanese a partire dal 2009. Tuttavia, per quanto riguarda il suo funzionamento, ha bisogno di ulteriori miglioramenti.

\section{Obiettivi imposti dalle direttive comunitarie in materia di energie rinnovabili}

Ai sensi dell'art.4 della direttiva 2009/28/CE, alle parti contrattuali del trattato CE è stato chiesto di avere, indicativamente, i seguenti obiettivi per quanto riguarda la parte dell'energia con fonti rinnovabili nel 2020: a) Riscaldamento e Raffreddamento; b) Energia; e c) Trasporto.

II calcolo degli obiettivi specifici di questi tre settori convertiti in predittori di valore energetico deve essere quanto grande quanto la quantità di energia prevista da fonti rinnovabili che corrispondono all'obiettivo generale di FER per l'Albania nel 2020, come è riportato nella cella finale della tabella 6b. Inoltre, l'obiettivo specifico per il trasporto deve essere in conformità con le richieste di cui all'art.3 della direttiva 2009/28/CE per un livello del 10\% della parte coperta da energie rinnovabili nel settore dei trasporti. Per quanto riguarda i prodotti petroliferi, solo il diesel e gasolio sono calcolati per il designatore.

Ciò significa che il carburante "Jet" utilizzato in aviazione, nonché il carburante utilizzato per le navi non viene calcolato. I biocarburanti dalla spazzatura, i rifiuti, la cellulosa, i materiali non alimentari e il materiale lignocellulosa sono calcolati il doppio per quanto riguarda il designatore; L'obiettivo generale nazionale per l'energia derivante da fonti rinnovabili per la produzione energetica lorda finale nel 2009 e nel 2020 è stata presentata nella tabella 1.

Tabella 1: Obiettivo Generale Nazionale

\begin{tabular}{|c|l|c|c|}
\hline A & ERE $^{3}$ parte consumata rispetto a EGFC 4 durante 2009-2014 & $\%$ & $30 \%$ \\
\hline B & Obiettivo ERE che verrà consumato in EGFC nel 2020 & $\%$ & $37 \%$ \\
\hline C & Quantità ERE consumato in media in EGFC durante 2009-2014 & ktoe & 667 \\
\hline D & EGFC per l'Albania durante il 2009-2014 & ktoe & 2,282 \\
\hline E & EGFC prevista per l'Albania nel 2020 (con EE) & ktoe & 2,678 \\
\hline F & ERE quantitativo annuo aggiuntivo per raggiungere l'obiettivo nel 2020 & ktoe & 397 \\
\hline G & Quantità totale annua ERE da consumare nel 2020 & ktoe & 1,017 \\
\hline
\end{tabular}

Il sostegno statale deve essere ristrutturato per le energie rinnovabili e va a sostituire lo schema di feed-in tariff con un sistema basato sui contratti per la differenza (CFD). In ogni caso, nella legislazione che viene modificata, il regime di feed-in tariff dovrebbe rimanere in vigore per sostenere l'energia rinnovabile in una piccola scala fino al MW di potenza installata per 2 unità di generazione.

I produttori di energia rinnovabile, aventi per il momento contratti di vendita sicura di energia prodotta, finalizzati prima della entrerà in vigore della legge che va modificata, avranno una tariffa fissa feed-in per la parte restante del contatto. Questa situazione non esclude il diritto di questi produttori di passare ad un regime di contratto non protagonista per la differenza.

\footnotetext{
${ }^{3}$ Enti Rregullator i Energjise

${ }^{4}$ Energy Gross Final Consumption
} 
Sulla base della legge 43/2015 sul settore energetico, il nuovo modello di mercato dell'energia è in corso di elaborazione e prevede la commercializzazione dell'energia da fonti rinnovabili, per un mercato avanzato appena si stabilisce il mercato dello stock energetico albanese.

I criteri legali della generazione RES sono stabiliti dall'ERE. La generazione che soddisfa le condizioni di legge potrebbe essere supportata solo in virtù di un CFD. Le tecnologie rinnovabili che saranno accettate per supportare lo schema CFD sono elencate nel paragrafo seguente: a) Trasformazione della biomassa; b) Vento in confini terrestri; c) Fotovoltaico solare; d) Hydro Energy; e) Energia dai rifiuti attraverso CHP; f) Gas delle discariche.

II MEI5 riesamina l'elenco ogni anno e potrebbe proporre una modifica alla lista in occasione del Consiglio dei Ministri nel caso in cui un'altra tecnologia sia considerata consolidata.

Inoltre, i cambiamenti legali forniranno per la redazione delle metodologie per la determinazione di prezzi standard calcolati in modo tale da sostenere la produzione di una notevole energia rinnovabile, economicamente accettabili dal mercato.

La legge sulle RES prevede che il Consiglio dei Ministri adotti politiche e misure per aumentare l'uso di energia solare negli edifici, compreso l'obbligo per le diverse categorie di edifici, nuovi o esistenti, pubbliche o private essi siano, al fine di installare sistemi di riscaldamento sfruttando l'energia solare per fornire acqua calda e tecnologica, quindi di conseguire gli obiettivi per aumentare l'immissione di energia solare nel consumo totale di energia all'interno del paese.

Lo stesso articolo impone al Consiglio dei Ministri, con l'approvazione del ministro responsabile per l'energia, di identificare i settori economici e le categorie di edifici, la superficie minima o la capacità del sistema di riscaldamento dell'acqua con l'energia solare, i requisiti tecnici dei sistemi di riscaldamento dell'acqua con energia solare che verrebbero installati, così come le procedure e i criteri specifici da seguire in connessione con l'attuazione e il monitoraggio di tale obbligo da parte delle istituzioni competenti.

II ministero responsabile per il settore della costruzione è il Ministero dello Sviluppo Urbano. Tuttavia, le questioni energetiche degli edifici sono divise in una certa misura tra il Ministero dello Sviluppo Urbano e il Ministero dell'Energia e dell'Industria. Vale la pena notare che, sulla base della normativa vigente in materia di pianificazione e sviluppo urbano, le autorità locali hanno un ruolo chiave nel settore delle costruzioni e sono responsabili per il rilascio dei permessi e il monitoraggio delle costruzioni.

La nuova legge 124/2015 in materia di efficienza energetica attribuisce più potere all'Agenzia responsabile per l'efficienza energetica per quanto riguarda il rendimento energetico degli edifici, dove la conformità di ciascun obiettivo obbligatorio per l'installazione RES negli edifici sarà monitorata dal Quadro delle prestazioni complessive dell'edificio.

La legge RES non stabilisce alcun termine in questa misura, tuttavia, un progetto di decisione governativa è già pronto, che istituisce un programma per l'installazione obbligatoria di sistemi NUED 6 in diverse categorie di edifici. E' importante che l'adozione e l'attuazione di questa decisione sia in linea con la nuova normativa in materia di rendimento energetico degli edifici (legislazione sull'efficienza energetica).

Una revisione importante della legislazione esistente è quella proposta dal Programma UNDP ${ }^{7}$ sui cambiamenti climatici per la creazione e il rafforzamento del mercato dei pannelli solari per l'acqua calda, e per la revisione della legge no.10112, datata 9.4.2009 "Sulla somministrazione di comproprietà in edifici residenziali ". Basandosi sulle revisioni proposte, il sistema di installazioni del NUED devono essere inclusi nelle comproprietà dei condomini che permettono un migliore funzionamento e gestione di questi sistemi.

In accordo con il programma UNDP sui cambiamenti climatici per la creazione e il rafforzamento di pannelli solari di mercato per l'acqua calda, è stata presentata una proposta in virtù di una decisione del consiglio comunale di Tirana per l'installazione all'interno di superfici minime di NUED in diverse categorie di edifici, tra cui edifici ad uso abitativo, condomini, uffici privati, alberghi e ristoranti, impianti sportivi.

Secondo la decisione del progetto, il sistema NUED avrà una superficie cui soddisfi almeno il $60 \%$ del fabbisogno di acqua calda, a parte le piscine aperte che dovrebbero riscaldare completamente l'acqua dal calore degli impianti solari termici.

Allo stato attuale, l'utilizzo di energie rinnovabili nei regolamenti edilizi non è regolato da un livello minimo. Come accennato in precedenza, la nuova legge sulle energie rinnovabili, impone al Consiglio dei Ministri di fissare obiettivi minimi per la produzione di acqua calda sanitaria e tecnologica utilizzando l'energia solare negli edifici e l'installazione

\footnotetext{
5 Ministero dell'Energia ed Industria

${ }^{6}$ Sistemi tecnologici in grado di fornire energia per il riscaldamento dell'acqua.

${ }^{7}$ Programma di Sviluppo delle Nazioni Unite
} 
obbligatoria di sistemi NUED per una categoria di edifici. Nel frattempo, un progetto decisivo del governo è preparato per l'installazione obbligatoria di sistemi NUED all'interno degli edifici. II progetto-decisione contiene anche la zona minima NUED che deve essere installata per soddisfare la domanda di costruzione per l'acqua calda. Secondo il progetto di decisione, il sistema NUED avrà un'area che dovrà soddisfare almeno il $60 \%$ della richiesta di acqua calda, a parte l'aggiunta che dovrà riscaldare completamente l'acqua da sistemi solari termici.

La politica del governo non ha preso in considerazione nessuno degli obblighi imposti per quanto riguarda i livelli minimi di energia da fonti rinnovabili nelle nuove e recenti edifici. Sulla base dei requisiti della nuova legge sulle energie rinnovabili, questi livelli minimi dovrebbero essere presi in considerazione da queste politiche.

Le informazioni attuali e future, nonché le campagne di sensibilizzazione e i programmi, insieme con le revisioni previste e i risultati attesi devono essere descritte per essere più chiari possibili. Gli Stati membri dovrebbero indicare quale autorità è responsabile del monitoraggio e rivedere gli effetti dei programmi. Per esempio, quando le autorità regionali, i governi hanno un ruolo chiave, bisogna specificare e riassumere il percorso dei lavori.

Ai sensi dell'art.8 della Legge sulle energie rinnovabili, il Ministero responsabile dell'energia e l'agenzia autorizzata, responsabile per le risorse energetiche rinnovabili, Agenzia Nazionale delle Risorse Naturali (NANR) assicura che tutte le parti interessate, come i clienti, gli sviluppatori, i progettisti, gli sponsor, gli investitori, le istituzioni finanziarie, i costruttori, gli installatori, gli architetti e i fornitori di apparecchiature e i sistemi di riscaldamento e raffreddamento, gli impianti di energia elettrica e altri dispositivi che utilizzano le energie rinnovabili, sono resi disponibili delle informazioni per supportare le misure sugli impianti che utilizzano fonti di energia rinnovabili.

Lo stesso articolo (comma 2) prevede che il Ministero responsabile per l'energia o l'agenzia responsabile per le energie rinnovabili dovrebbero sviluppare programmi adeguati di formazione o di orientamento sulla sensibilizzazione e informazione del pubblico, al fine di informare le parti interessate e i cittadini sui benefici, sui costi e sulla realizzazione dello sviluppo e l'utilizzo di energia derivabile da fonti rinnovabili.

La MEI o le istituzioni subordinate non hanno fornito regole o procedure dettagliate sul modo di come fornire questa informazione. Tuttavia, va notato che si sono avviate una serie di campagne di sensibilizzazione da parte delle istituzioni di energia in Albania per promuovere l'uso delle FER in paese, attraverso vari seminari, pubblicità nei mass media ecc. MEI, e le sue istituzioni subordinate, hanno organizzato una serie di attività promozionali che gli investitori privati facciano fruttare prodotti di piccole dimensioni ma con ottimi risultati. Negli ultimi anni, un gran numero di investitori privati hanno mostrato vivo interesse a investire in energia elettrica prodotta da acqua e vento.

Non c'è ancora un piano di misure per promuovere l'uso di fonti energetiche rinnovabili a livello locale. Le nuove riforme territoriali, che saranno in grado di consentire la creazione di grandi comuni, le quali si aspettano emergano dopo le elezioni locali di giugno 2015 e possono essere un'ottima occasione per valorizzare il ruolo degli enti locali per l'attuazione delle politiche RES.

Come accennato in precedenza, il nuovo progetto sulle energie rinnovabili richiede, menzionando le informazioni a sostegno delle misure per l'utilizzo di fonti rinnovabili per l'energia elettrica, che il governo metta a disposizione queste informazioni per gli investitori, non solo attraverso la legislazione pubblicata nella Gazzetta ufficiale o vari siti web, ma anche attraverso una serie di eventi pubblici come conferenze, seminari, ecc.

Il governo mira a trasformare l'Agenzia responsabile per le Energie Rinnovabili in un centro di informazione per tutti gli stakeholders interessati in misure di sostegno per l'utilizzo di energie rinnovabili.

Al momento, nessuna istituzione o agenzia è responsabile dello sviluppo e nessuno fornisce orientamenti per gli urbanisti e gli architetti per aiutarli a tenere conto della combinazione ottimale delle risorse energetiche rinnovabili, tecnologie ad alta efficienza, così come riscaldamento e il raffreddamento durante la pianificazione, la progettazione, la costruzione e ristrutturazione di aree industriali o residenziali.

II governo Albanese sta preparando una nuova legislazione sul rendimento energetico degli edifici, comprese le disposizioni specifiche per la combinazione ottimale delle risorse energetiche rinnovabili e delle tecnologie ad alta efficienza nella pianificazione, progettazione, costruzione e ristrutturazione di aree industriali o residenziali.

I programmi di informazione, sensibilizzazione e formazione sui benefici e sulle pratiche di utilizzo di energie rinnovabili non sono stati organizzati adeguatamente. Le istituzioni responsabili per la politica energetica, soprattutto per il sostegno dei donatori internazionali, hanno lanciato una serie di campagne di informazione e sensibilizzazione ottenendo però scarsi risultati. L'unica area in cui questi programmi hanno avuto risultati soddisfacenti si riscontra nei programmi di promozione dei produttori di piccola potenza. II Ministero responsabile per l'Energia e l'Agenzia nazionale delle risorse naturali hanno mostrato un forte interesse nei confronti della promozione di impianti a pannelli solari per l'acqua calda a livello locale. 


\section{Valutazione dell'impatto attuativo della direttiva sull'efficienza energetica 2012/27/CE}

Albania, d'ora in poi non può permettersi di sprecare energia. L'obiettivo del governo Albanese è di riuscire ad avere un'energia efficiente tramite l'utilizzo ridotto del carbonio, il quale sarà anche sostenuto dagli aiuti dello stato e sarà in linea con gli obiettivi dell'efficienza energetica dell'Unione Europea.

Nel 2011 il governo albanese ha approvato due obiettivi energetici per il 2020 - ridurre le emissioni di gas serra, aumentando la quota di energie rinnovabili al 38\% e raggiungere l'efficienza energetica del 15\%. Questi obiettivi sono stati riconfermati dal Segretario della Comunità sull'Energia e sono condivisi tra le istituzioni europee, le quali hanno compito di assicurarsi che l'UE prenda le azioni necessarie per raggiungere gli obiettivi di Europa 2020.

Delle previsioni su EE nel 2011 hanno dimostrato che l'obiettivo dell'efficienza energetica 2020 non sarebbe stato soddisfatto ed è stato necessario adottare nuove misure a livello nazionale. È stata fatta una nuova proposta, in forma consolidata tra le parti contraenti nel segretariato comunitario sull'energia, volta a soddisfare la direttiva sull'efficienza energetica del 2012/27/UE. La proposta si basa sull'esperienza acquisita in diversi settori, in particolare sul rendimento energetico degli edifici e dei servizi energetici.

Per quanto riguarda la direttiva sull'efficienza energetica (EED) del 2012, I'Albania ha elaborato il suo recepimento parziale dal giugno 2014 e in questi giorni la decisione del governo albanese passerà in consegna al parlamento. Sostanzialmente, il recepimento parziale porta all'unione legale, a delle misure giuridicamente vincolanti nell'utilizzo dell'energia in modo efficiente in tutte le sue fasi della catena energetica - dalla trasformazione dell'energia e la sua distribuzione fino al consumo finale. I requisiti più importanti della direttiva, in termini di futura politica energetica, sono riportate di seguito:

1) In primo luogo, al fine di rafforzare l'impegno politico da parte del governo albanese nella strategia UE 2020 , la EED definisce chiaramente e quantifica l'obiettivo di efficienza energetica vero e proprio:

Il consumo energetico albanese previsto per il 2020 è non superiore a 2.420 ktep di energia primaria o non più di 2.045 ktep di energia finale a confronto con il 2013. La realizzazione parziale e corretta della direttiva del 2012 avrà un ruolo importante nel raggiungere almeno il 10\% di efficienza energetica e un risparmio di almeno $350 \mathrm{ktep}$ fino al 2020.

Lo scenario dell'efficienza energetica rappresenta il potenziale di risparmio energetico dell'Albania adottando le attuali tecnologie disponibili per migliorare la sua efficienza. Si presume che una serie di politiche migliori per l'uso energetico siano utilizzate in tutti i settori, con conseguente notevole riduzione del consumo di energia (per esempio, apparecchiature più efficienti elettrici nel settore domestico, il miglioramento termico dell'integrità degli edifici, uso di processi più efficienti nel settore industriale, etc.).

L'applicazione di queste misure si presume iniziasse intensamente nel 2015. Nel 2020 vi si aspetta un risparmio energetico del $10 \%$ del consumo finale di energia rispetto allo scenario di base, mentre nel 2030 si attende un risparmio energetico del $20 \%$ del consumo finale di energia rispetto allo scenario di riferimento. Inoltre vi si aspettano degli investimenti intensivi per le reti nello scenario $\mathrm{EE}$, con la conseguente riduzione delle perdite di trasmissione e distribuzione al 12\% della produzione totale di energia elettrica nel 2030, rispetto al 22\% nel 2013.

Lo scenario EE, come attività principale per la strategia energetica del governo dell'Albania, secondo l'art.3 dell'EED contribuirà allo stabilire un obiettivo di risparmio del 20\% che potrebbe essere appropriato per il 2030.

L'impatto finanziario è l'obiettivo che EE per il 2020 (10\%) ha per l'Albania per il 2020 (10\%) ovvero di almeno 60 milioni di euro, invece per il 2030 più di 250 milioni di euro.

Per quanto riguarda le esigenze dell'art.5 sul Piano d'Azione per l'Efficienza Energetica Nazionale Albanese (NEEAP) è previsto che i governi centrali rinnovino ogni anno il $3 \%$ della superficie totale degli edifici di loro proprietà che non soddisfano i requisiti minimi di efficienza stabiliti dalla direttiva 2010/31/CE sul rendimento energetico nell'edilizia ad almeno i livelli di efficienza fissati dalla stessa direttiva.

Anche il NEEAP prevede di rinnovare almeno il 10\% del patrimonio edilizio del governo locale seguendo le misure di EE. L'Albania vuole mettere a disposizione di tutti gli edifici pertinenti governativi centrali e locali un inventario, entro il 31 dicembre 2015. L'ammontare di risparmio energetico richiesto sotto l'approccio alternativo è cumulativo, il che significa che l'Albania riesca ad ottenere un risparmio energetico annuo per l'intero periodo tra il 2014 e il 2020 fino al 4\% del capitale di destinazione.

Anche in NEEAP si assume la direttiva sugli appalti pubblici del 2004/18/CE, in linea con l'art.6 per i requisiti dell'EED, che i governi centrali e locali acquistano prodotti, servizi ed immobili ad alta efficienza energetica.

Per quanto riguarda l'art.7 della EED, I'Albania ha elaborato in EE una nuova legge per stabilire regimi obbligatori di efficienza energetica o utilizzare le misure di politica RES per raggiungere un importo mirato di risparmio energetico tra un numero limitato di "grandi" consumatori finali. 
I risparmi energetici da realizzare imposti dagli schemi obbligatori di efficienza energetica e dal RES sono ricalcolati per avere risparmi ogni anno dal 1/11/2015 al 31/12/2020 del 1.5\% delle vendite annue di energia per $\mathrm{i}$ consumatori finali di tutti i distributori di energia o di tutte le aziende consumatrici di energia in volume (250 MWh / anno) medio nel 2012, 2013 e 2014, con alcune possibilità specifiche, vale a dire utilizzando tassi di risparmio più bassi, parzialmente escludendo le industrie ETS, consentendo un sicuro risparmio dal lato dell'offerta o contando su un risparmio energetico dato da un'azione tempestiva a partire dal 31 dicembre 2014.

Secondo gli stabilimenti meridionali, l'Albania, in linea con l'art.8, impone la disponibilità di audit energetici tra i clienti finali in tutti i settori e garantisce che le imprese, che non sono PMI, effettuano audit energetici almeno una volta ogni 2 anni. Le verifiche sono assunte per essere redditizie e sono intraprese da esperti accreditati / qualificati o sotto la supervisione di autorità indipendenti.

La misurazione è uno dei problemi principali che emerge dal recente disegno di legge EE. È chiaro che l'art.9 non richiede l'introduzione di sistemi di misurazione intelligenti (questo è trattato nelle direttive del Terzo pacchetto: 2009/72/CE e 2009/73/CE), piuttosto chiarisce se una parte di energia elettrica di DSO introduce sistemi di misurazione intelligenti, diversi obblighi che devono applicarsi secondo i requisiti della direttiva, ad esempio, che i contatori intelligenti devono essere in grado di misurare l'elettricità immessa in rete dalla sede al cliente. Si stabilisce inoltre che la valutazione del "cliente finale" potrebbe non includere solo la persona che utilizza l'energia, ma anche una persona 0 un'organizzazione come una cooperativa di proprietari in un edificio di più appartamenti, che acquista energia collettiva.

L'Albania è un paese che utilizza energia elettrica in vasta gamma, a fini di riscaldamento e raffreddamento. In questo senso la politica energetica del paese ha lo scopo di preparare una valutazione globale per identificare il potenziale economico del riscaldamento efficiente e il potenziale del raffreddamento ad aria/geotermica, tenendo conto delle condizioni climatiche, della fattibilità economica e idoneità tecnica. Questa valutazione è già notificata dall'atto della legge subordinata preparato durante il 2015.

\section{Conclusioni}

In conclusione, per far in modo che l'obiettivo del consumo nazionale di ERE-T del 38\% per essere realizzato nel 2020, è stato previsto un consumo del $3 \%$ per il territorio albanese di EGFC. Altrimenti, è stata prevista anche la miscela nella misura del $10 \%$ in volume di combustibili dei materiali rinnovabili (FAME), consumata nel settore dei trasporti. Tale importo sarà pari ad almeno al 75 ktep di FAME per il settore dei trasporti nel 2020. Questo importo della parte rinnovabile in biocarburanti prende in considerazione l'aumento di 60 ktoe nel 2020 rispetto al 2014.

Inoltre, affinché l'obiettivo del consumo nazionale dell'ERE-H\&C del 38\% che dev'essere realizzato nel 2020, è necessario che si consumi il 10\% del EGFC nel territorio albanese, speso per riscaldamento e raffreddamento. Questo importo implica un'ulteriore aumento del calore con almeno 800 GWh/anno nel 2020 e un consumo annuo di ERE-H \& C di 2/9 di calore ktep dal legno/industriale/rifiuti e pompe di calore di biomassa.

Allo stesso tempo, che si raggiunga il consumo del 38\% di ERE-H\&C nel 2020, è necessario che si consumi la quantità del 29\% di EGFC, utilizzato nel settore energetico in territorio albanese per il riscaldamento e il sistema di raffreddamento. Questo importo implica un ulteriore aumento di almeno 270 ktep (3.140 GWh) fino al 2020. Inoltre, questo significa anche un aumento medio dei generatori di energia da fonti rinnovabili per un importo di 830 MW (20-35\% fattore di capacità).

Attualmente, non ci sono organi responsabili a fornire informazioni sull'uso di energie rinnovabili nel settore del riscaldamento e raffreddamento e nel settore dei trasporti. Come accennato in precedenza con la nuova legge sulle energie rinnovabili (l'articolo 25), il Consiglio dei Ministri emana un regolamento per garantire un'informazione pubblica sulle proprietà e i benefici per l'ambiente che derivano da tutte le diverse fonti di energia rinnovabile per i trasporti. II regolamento dovrebbe inoltre prevedere l'obbligo di indicare nei punti vendita in cui il contenuto di biocarburanti diluiti in olio da prodotti superino il limite impostato in percentuale, in base alle richieste effettuate dall'ERE.

Negli ultimi dieci anni, la maggior parte del dibattito sulla promozione delle fonti rinnovabili è stata focalizzata sui regimi di sostegno finanziario e sul miglioramento delle condizioni di accesso alla rete per l'elettricità da fonti rinnovabili. Senza dubbio, si tratta di questioni essenziali che continueranno ad essere al centro dell'attenzione dei policy makers attuali e futuri. Tuttavia, nel corso degli ultimi anni, "l'importanza di individuare e affrontare le barriere non-finanziarie e di natura non tecnica, l'elettricità rinnovabile ha attirato l'attenzione di analisti e di governi". Quelle sono altre aree importanti che la NREAP ha focalizzato la sua attenzione.

In conclusione, l'efficienza energetica è una questione complessa ed esigente in termini capacità di costruzione ed esecuzione delle politiche di governo per l'Albania. Politicamente, c'è un divario tra gli impegni assunti dall'Albania dal 
2011 e la relativa consegna. La direttiva 2012/27 EE dà una nuova struttura sovra-legale per gli obiettivi di efficienza energetica concordati a livello europeo e dal segretariato della Comunità dell'energia. Fino ad oggi, per lo stato Albanese, l'applicazione della legislazione esistente è di parziale efficienza energetica e molto limitata.

L'EED sostituisce e rafforza l'efficienza della direttiva sull'energia del 2006/32/CE sui servizi energetici e i collegamenti con gli obblighi già stabiliti nella direttiva 2009/125/CE sull'Eco-design, la direttiva 2010/30/UE sull'etichettatura energetica e la direttiva 2010/31/UE sulla prestazione energetica degli edifici. Come accennato in precedenza, il governo albanese con il ministero dell'Energia e dell'Industria ha riferito nel luglio 2015 i progressi verso gli obiettivi del 2020, e questa valutazione può includere proposte di ulteriori misure necessarie per l'attuazione della nuova legge EE per soddisfare i requisiti parziali del 2012/27/UE.

\section{References}

Bollettino ufficiale: "Piano d'Azione Nazionale Albanese per le Energie Rinnovabili" (NREAP), Governo Albanese, 2015.

Bollettino ufficiale: "Piano d'Azione Nazionale Albanese per l'Efficienza Energetica" (PNAEE), Governo Albanese, 2014

Legge n. 43/2015, del 2015/04/30 "On settore energetico".

Legge n.125 / 2013 del 25.04.2013 "Sulle concessioni e partenariato pubblico-privato".

Legge n.111/, del 15.11.2012 "Sulla gestione integrata delle risorse idriche".

Legge n. 138/2013 del 02.05.2013 "Sulla risorse energetiche rinnovabili".

Legge n.10112, del 9.4.2009 "Sulla somministrazione di comproprietà in edifici residenziali".

EU Directive 2009/28/EC "On the enhancement of the use of energy from renewable resources".

EU Directive 2012/27/EU "On energy efficiency".

https://ec.europa.eu/energy/en/topics/, 06/2016.

http://www.energjia.gov.al/, 06/2016.

http://www.akbn.gov.al/category/energjite-e-rinovueshme/, 06/2016. 